\title{
Health care-associated infections studies project: An American Journal of Infection Control and National Healthcare Safety Network data quality collaboration case study: Bloodstream infection-patient injection into vascular access 2018
}

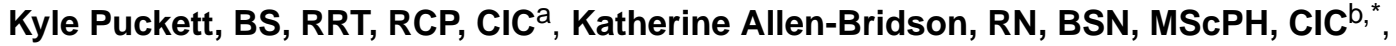 \\ Dominque Godfrey, MPH, CPH, CIC ${ }^{c}$, Cindy Gross, MT, SM(ASCP), CIC ${ }^{\mathrm{a}}$, Joan N. Hebden, \\ RN, MS, CIC $^{d}$, Latasha Powell, RN, BSN, MPH, CIC ${ }^{a}$, and Marc-Oliver Wright, MT(ASCP), \\ MS, $\mathrm{CIC}^{\mathrm{e}}$ \\ ${ }^{\mathrm{a} C A C l}$, Atlanta, GA \\ ${ }^{b}$ National Healthcare Safety Network, Division of Healthcare Quality Promotion, Centers for \\ Disease Control and Prevention, Atlanta, GA \\ 'Leidos, Reston, VA \\ dIndependent Infection Prevention Consultant, Baltimore, MD \\ eDepartment of Infection Control, University of Wisconsin Hospitals and Clinics, Madison, WI
}

\begin{abstract}
This case study is part of a series centered on the Centers for Disease Control and Prevention/ National Healthcare Safety Network (NHSN) health care-associated infection surveillance definitions. These cases reflect some of the complex patient scenarios infection preventionists have encountered in their daily surveillance of health care-associated infections using NHSN definitions and protocols. Teaching points for this case study are:

- Device day counts for denominator data

- $\quad$ Eligible central line (CL) day counts for device attribution

- $\quad$ Associating bloodstream infections with CLs

- Documentation required for use of the NHSN CL- associated bloodstream infection exclusion for observed
\end{abstract}

\footnotetext{
*Address correspondence to Katherine Allen-Bridson, RN, BSN, MScPH, CIC, National Healthcare Safety Network, Division of Healthcare Quality Promotion, Centers for Disease Control and Prevention, 1600 Clifton Rd, MS A-24, Atlanta, GA 30333., kabridson@comcast.net (K. Allen-Bridson).

Conflicts of interest: None to report.

Note: NHSN BSI protocol states, "A BSI meeting LCBI criteria that is accompanied by documentation of observed or suspected patient injection into the vascular access line, within the BSI Infection Window Period, will be considered an LCBI but not a CLABSI for NHSN reporting purposes."
} 


\section{Keywords}

Intravenous injection; Central line-associated bloodstream infection; NHSN

This case study is a continuation of a published series in the American Journal of Infection Control. These cases reflect the examples of complex patient scenarios infection preventionists encounter in the daily surveillance of health care-associated infections using National Healthcare Safety Network (NHSN) definitions. Objectives have been published previously. ${ }^{1}$

With each case, there is a link to an online survey, where you may answer the questions and receive immediate feedback in the form of answers, explanations, and references. Individual participant answers will remain confidential, although the authors intend to share a summary of aggregated findings at a later date. The case scenario, answers, and explanations have been reviewed and approved by NHSN Protocol Validation and Training team staff. We hope you will take advantage of this educational opportunity by actively participating, and we look forward to providing you with additional case studies. The online survey can be found at https://www.surveymonkey.com/r/BSI-PatientInjectionintoVascularAccess.

We strongly recommend that participants review or reference the NHSN Patient Safety Component Manual for information that may be needed to answer the case study questions. The Web site links pertinent to this case study are https://www.cdc.gov/nhsn/pdfs/ pscmanual/2psc_identifyinghais_nhsncurrent.pdf and https://www.cdc.gov/nhsn/pdfs/ pscmanual/4psc_clabscurrent.pdf.

The findings and conclusions of this case study are those of the authors and do not necessarily represent the official position of the Centers for Disease Control and Prevention.

For each question, please select the most correct answer.

\section{Scenario:}

On January 1, 2018, a 23-year-old man with paraplegia secondary to T1 transverse myelitis is admitted to an inpatient medical ward. An implanted port and suprapubic catheter are present on admission, both having been placed in June 2017. The unaccessed port is scheduled for removal January 3 because of a history of recurrent polymicrobial bacteremia. The most recent bloodstream infection (BSI) occurred during a previous admission in late November, and the patient completed a 4-week course of antibiotics on December 31. Blood cultures in November were positive for Candida albicans, Chryseobacterium indologenes, and Enterococcus faecalis.

On January 3 at 12:23 AM (hospital day 3), the patient began complaining of "itching at port insertion site." Documentation in the chart states, "Patient is scratching port site." Port insertion site is red, warm, and tender to touch. The port is accessed for the first time during this admission to collect blood cultures. One set is collected from the port and one from a peripheral venipuncture. The port is deaccessed after specimen collection, and a previously 
planned port removal is postponed pending blood culture results. A peripheral intravenous line is placed for temporary access.

On January 4, blood cultures collected on January 3 are preliminarily resulted as no growth. The port is removed and the patient sleeps most of the afternoon.

On January 5, the patient pulls his intravenous line out. A right upper arm peripherally inserted central catheter (PICC) is inserted at 1:30 PM, with verification of proper placement. Chart documentation shows the following: "Patient continues to be noncompliant with medical care (specifically, refusing activities of daily living, wound care, and medications except narcotics). The nurse witnessed patient tampering with PICC (specifically, disconnecting the continuous infusion frequently against medical advice, picking at the dressing, and scratching aggressively around the new PICC insertion site)." Lowgrade fever of $99.8^{\circ} \mathrm{F}$ is noted at 8:00 PM.

On January 6 at 11:00 AM, the patient complains of pain, severity 10/10, and oxycodone 15 $\mathrm{mg}$ is given. The patient is requesting to leave the unit to smoke with visitors. The registered nurse advises against it since he just took pain medicine, and the patient agrees to wait half an hour but then insists on leaving at 11:20 AM. The patient is alert and oriented, and the central line (CL) is disconnected and capped by the nurse so the patient can leave the floor. At 12:40 PM, the patient returns to the unit, after being gone for over an hour, slurring words and unable to keep his eyes open. He appears very sleepy, with a marked change in level of consciousness since leaving the floor. The safety cap is missing from the secondary port, and the CL is unclamped. The patient responds, "I don't know," when asked about the condition of the CL. The physician is informed of events and arrives for a patient assessment at 1:30 PM. The physician agrees with the patient assessment and notes the patient's current condition is inconsistent with current narcotic orders and previous response to administration of ordered pain medications. She orders discontinuation of the PICC and all narcotics. At 5:15 PM, the right upper arm PICC is removed and the patient is more alert but very unhappy about removal of the CL and discontinuation of narcotics.

On January 7 , the patient spikes a fever of $101.2^{\circ} \mathrm{F}$, and the white blood cell count is

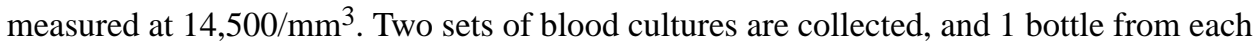
set is positive for Staphylococcus hominis, Klebsiella oxytoca, and Enterococcus faecium. The blood cultures collected on January 3 are still showing no growth (See Table 1: Refer to the following reference table as needed to answer questions).

Question 1: On January 7, how many CL days have occurred to determine if the BSI is a CL-associated BSI (CLABSI)?
a. $\quad 6$ CL days
b. 4 CL days
c. 2 CL days
d. None; the BSI is not CL-associated

Question 2: What is the correct determination for the positive blood specimens collected January 7, and how should the field for CL be completed? 
a. Laboratory-confirmed bloodstream infection (LCBI) 1 ; $\mathrm{CL}=$ No because of self-injection, date of event (DOE) January 7

b. LCBI 1; CL = Yes (CLABSI), DOE January 7

c. $\quad$ LCBI 2; CL $=$ No because of self-injection, DOE January 5

d. $\quad$ LCBI $2 ; \mathrm{CL}=$ Yes (CLABSI), DOE January 5

e. No LCBI because patient is noncompliant and tampering with line

Scenario continued:

On January 8, a left upper arm PICC is placed for treatment of polymicrobial bacteremia. Repeat blood cultures are collected. A personal sitter is assigned to the patient, but no reason for this order is documented in the patient's chart.

On January 9, the blood cultures from January 8 are positive for $E$ faecium and Pseudomonas aeruginosa.

On January 11, the physician progress note states, "Patient with continued noncompliant behavior despite repeated attempts to explain the serious health consequences. Recurrent polymicrobial bacteremia, with workup negative for a focus of infection. In light of his past medical history of significant substance abuse, likely represents ongoing contamination of CL from patient injecting illicit drugs. It is in the patient's best interest to discontinue the CL and change to oral antibiotics. Left upper arm PICC removed at 2:40 PM."

On January 13, repeat blood cultures are collected, and all are negative for growth. A physician progress note states, "PICC removed January 11; recent blood cultures clear."

Question 3: Which statement about counting device days for reporting January CL summary denominator data is correct?

a. Total device days reported for this patient for January CL summary denominator data: 6 device days

b. Total device days reported for this patient for January CL summary denominator data: 8 device days

c. Total device days reported for this patient for January CL summary denominator data: 9 device days

d. Total device days reported for this patient for January CL summary denominator data: 10 device days

Question 4: Which of these documented notes would meet criteria for use of the patient self-injection exclusion, assuming LCBI criteria are met and documentation is within the BSI window period?

1. "Patient is manipulating his CL by scratching around it aggressively and interfering with line maintenance by refusing care."

2. "Patient is very noncompliant with medical care (refusing activities of daily living, wound care, CL care, and medications except narcotics). Have 
witnessed patient tampering with PICC (disconnecting tubing to go outside to smoke with friends, picking at dressing, scratching aggressively around insertion site)."

3. "At 11:00 AM, patient complaining of pain, 10/10, and oxycodone $15 \mathrm{mg}$ given per medication administration record. Several friends came to visit, requesting to go out to smoke. Registered nurse advised against it since he just took pain medicine. Patient agreed to wait half an hour but insisted on leaving at 11:20 AM; patient off the floor with friends. Patient back on unit at 12:40 PM (gone for over an hour), slurring words, with difficulty keeping eyes open, and appears very sleepy. Vital signs per flow sheet. Safety cap was missing from the secondary port, and the line was unclamped. Physician informed of events."

4. "Changed to oral antibiotics because of misuse and contamination of intravascular line."

5. "Patient with continued polymicrobial bacteremia, with workup negative for a focus of infection. In light of his past medical history of significant substance abuse, likely represents contamination of CL from patient using line to inject unknown substance. CL removed, and recent blood cultures are clear."
a. $\quad 1,2$, and 4
b. $\quad 1$ and 4
c. 3 only
d. 5 only
e. All of the above

\section{Reference}

1. Wright MO, Hebden JN, Bridson KA, Morrell GC, Horan T. Healthcare-associated Infections Studies Project: An American Journal of Infection Control and National Healthcare Safety Network Data Quality Collaboration. Am J Infect Control 2010;38:416-8. [PubMed: 20583335] 


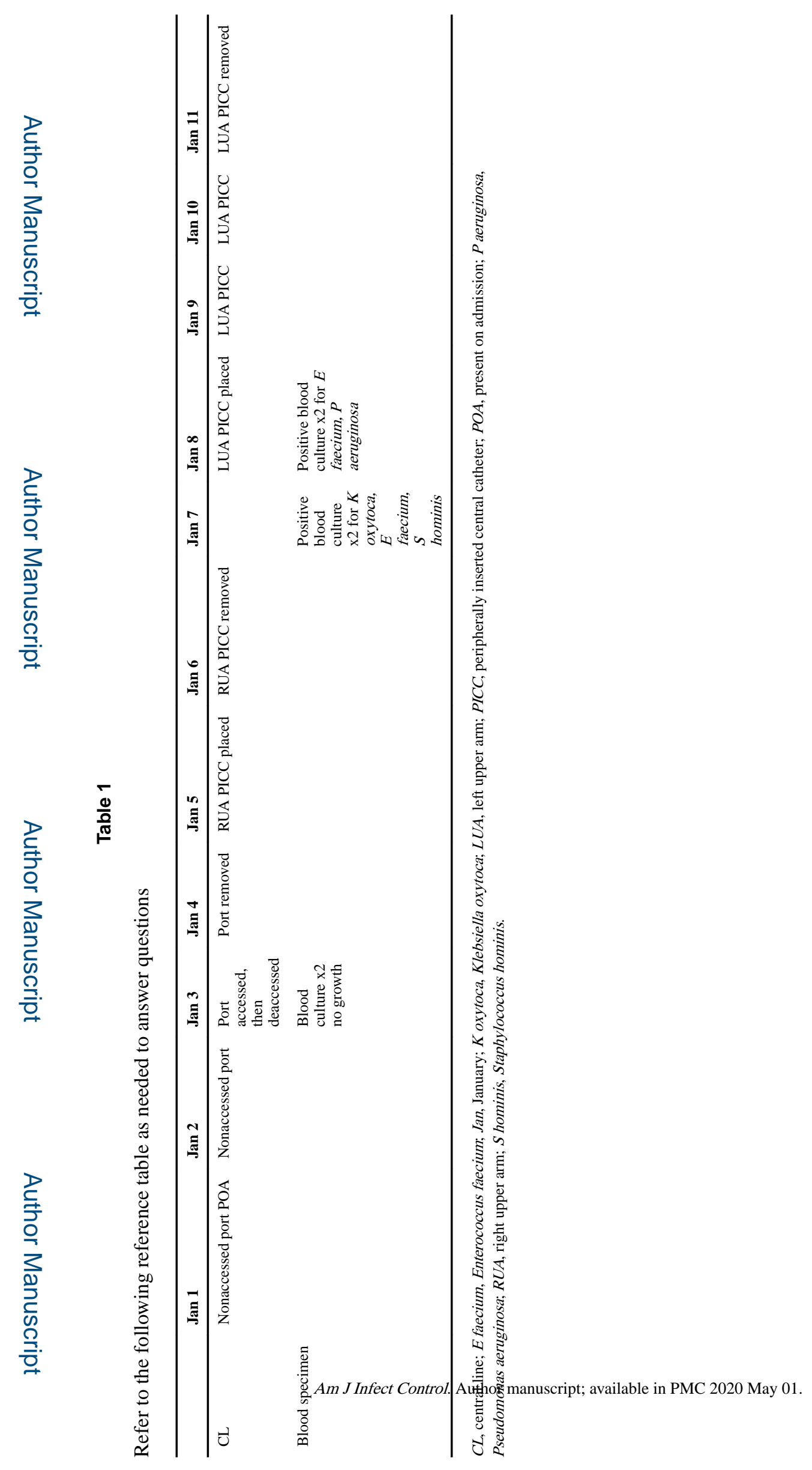

